\title{
Landing Autopilot for the Control of Airplane by Using the $\mathrm{H}$-inf Control
}

\author{
Mihai Lungu and Romulus Lungu
}

\begin{abstract}
The paper focuses on automatic control of airplane in longitudinal and lateral-directional planes, during landing, by using the linearized dynamics of airplane and the $\mathrm{H}$-inf control; both planes (longitudinal and lateral-directional) are treated; the new obtained automatic landing system will consist of three subsystems: the first two control airplane's motion in longitudinal plane, while the third one is used for the control of airplane motion in lateral-directional plane. The theoretical results are validated through numerical simulations for a Boeing 747 airplane.
\end{abstract}

Index Terms - Landing, autopilot, H-inf control.

\section{INTRODUCTION}

During landing (most difficult stage of flight), the airplanes have to perform a precise maneuver in the proximity of the ground to land safely at a suitable touch point with acceptable sink rate, speed, and attitude [1]. There are many methods to design automatic landing systems (ALSs); for the control of the lateral motion during landing, an Instrumental Landing System (ILS) type radio-navigation system together with a system used for the obtaining of distances between the airplane and the runway radio-markers has been used in [2], [3]. The direction controllers which are used within such ALSs can be PD, PI, or PID type, in classical or fuzzy variants [4] etc. In the research area of optimal synthesis, Ochi and Kanai have used the H-inf control technique to design airplanes' automatic approach and landing [5], but the authors did not analyze the robustness of the designed controllers in the presence of sensor errors and wind shears. The usage of different adaptive control architectures such as the ones based on the dynamic inversion technique and neural networks (NNs), with or without Pseudo Control Hedging blocks [6] is motivated by the presence of known nonlinearities associated to the dynamics of airplane or actuators as well as to the external disturbances. In [7] some feed-forward neural networks based on the back propagation learning algorithm have been used, but their main disadvantage is related to the priori training on normal and faulty operating data. The main disadvantages of the NN based approaches designed in $[8,9]$ are the required operating conditions which are not easy to satisfy in practical control applications. On the other hand, the main drawback of all the papers dealing with airplane landing is that the designed ALSs are designed either

Manuscript received on January 2, 2017; revised May 1, 2017. This work is supported by grant no. 89/1.10.2015 of the Romanian National Authority for Scientific Research and Innovation, CNCS-UEFISCDI, code PN-II-RU-TE-2014-4-0849.

Mihai Lungu and Romulus Lungu are with University of Craiova, Faculty of Electrical Engineering, Craiova, Romania (e-mail: Lma1312@yahoo.com, romulus_lungu@yahoo.com). for the longitudinal plane or for the lateral-directional plane. The present work focuses on airplane automatic control in the two planes, during landing, by using the $\mathrm{H}$-inf control. Our aim is to design a new landing control system (both planes) which cancels the negative effect of possible disturbances (wind shears, crosswind, or errors of the sensors).

\section{CONTROL OF THE AIRPLANE IN LONGITUDINAL PLANE}

The landing procedure involves three main phases: initial approach, glide slope, and flare [10]. The initial approach means the descent of airplane from the cruise altitude to approximately $420 \mathrm{~m}$ above the ground. During next two stages, the speed and pitch angle must be controlled; as airplane descends to 7-20 $\mathrm{m}$ (the maximum value is for Boeing 747), the slope angle control system is disengaged and a flare maneuver is executed [11]. The vertical descent rate is slightly decreased such that the landing gear may be able to dissipate the energy of the impact at landing. The pitch angle of the airplane is then adjusted, between 0 to 5 degrees to allow a soft touchdown on the runway surface [12]. These issues will be achieved by the first two systems presented in this paper - the ones for the control of airplane's trajectory in the longitudinal plane. Airplane motion in lateral plane should be done without errors, this meaning the cancel of airplane's deviation with respect to the runway direction; for this, there will be designed the third system presented in this work - the one for the control of airplane trajectory in the lateral- directional plane. In Fig. 1, there are presented the main elements of airplane's geometry with respect to the runway - longitudinal and lateraldirectional planes; in Fig. 1, PA is the glide slope, PDA - the runway, $\gamma_{c}-$ the imposed slope angle of the trajectory, $R-$ the distance between airplane and the radio-marker, $\gamma-$ the slope angle, $Y$ - the airplane lateral deviation with respect to runway, $\bar{\psi}-$ the runway direction, $\psi-$ the flight direction, $\beta-$ the glide slope angle. The expression of the angle $\Gamma$ is [13]:

$$
\Gamma=\frac{1}{R} \int_{0}^{t} V \Gamma^{\prime} \mathrm{d} \tau=\frac{1}{R} \int_{0}^{t} V\left(\gamma_{c}-\gamma\right) \mathrm{d} \tau \cong \frac{V_{0}}{R} \int_{0}^{t} V\left(\gamma_{c}-\gamma\right) \mathrm{d} \tau .
$$

The linearized dynamics used in this paper for airplane's motion in longitudinal plane belongs to a Boeing 747 [13]:

$$
\dot{x}=A x+B u, y_{e}=C x,
$$

with $\boldsymbol{x} \in R^{6 \times 1}-$ the state vector, $x=\left[\begin{array}{llllll}V_{x} & V_{z} & \omega_{y} & \theta & \delta_{p} & \delta_{T}\end{array}\right]^{\mathrm{T}}$ and $u=\left[\begin{array}{ll}\delta_{p_{c}} & \delta_{T_{c}}\end{array}\right]^{\mathrm{T}}-$ the control input vector; $V_{x}$ is the longitudinal velocity, $V_{z}-$ the vertical velocity, $\omega_{y}-$ the pitch angular rate, 
$\theta-$ the pitch angle, while $\delta_{p}$ and $\delta_{T}$ are the elevator and the engine's command, respectively. The actuators are described

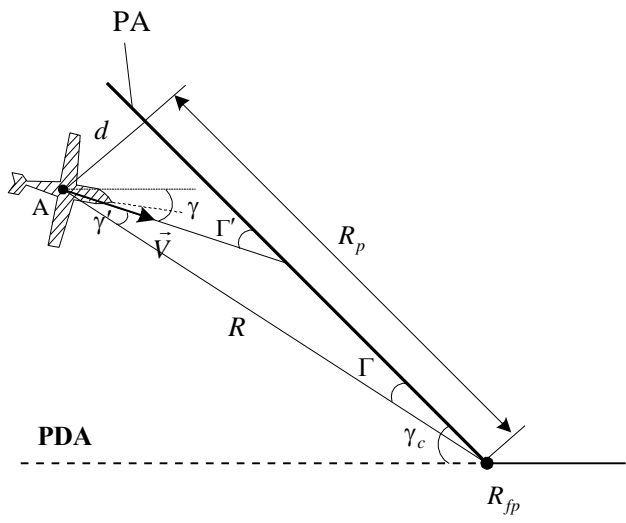

a.

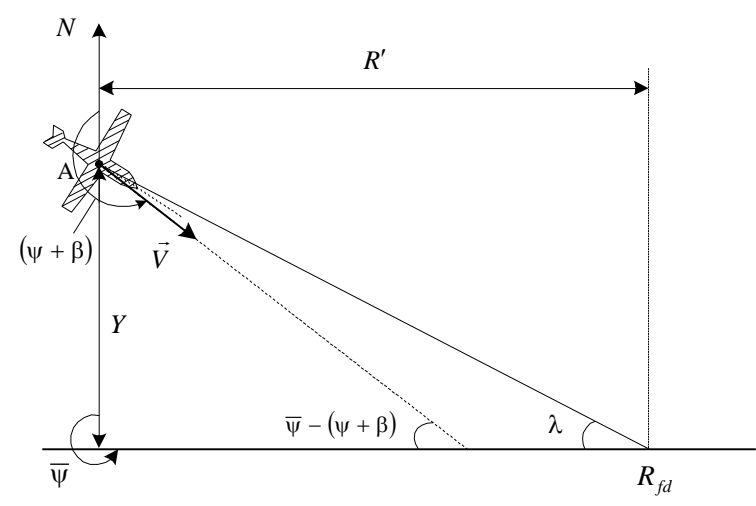

b.

Fig. 1. The geomtery of motions: a) longitudinal plane; b) lateral-directional plane.

by the equations: $T_{p} \dot{\delta}_{p}+\delta_{p}=\delta_{p_{c}}$ and $T_{T} \dot{\delta}_{T}+\delta_{T}=\delta_{T_{c}}$; $T_{p}, T_{T}$ are the elevator and engine's time constants. The forms of the matrices $A$ and $B$ are [13]:

$$
A=\left[\begin{array}{cccccc}
a_{11} & a_{12} & a_{13} & a_{14} & b_{11} & b_{12} \\
a_{21} & a_{22} & a_{23} & a_{24} & b_{21} & b_{22} \\
a_{31} & a_{32} & a_{33} & a_{34} & b_{31} & b_{32} \\
0 & 0 & 1 & 0 & 0 & 0 \\
0 & 0 & 0 & 0 & -\frac{1}{T_{p}} & 0 \\
0 & 0 & 0 & 0 & 0 & -\frac{1}{T_{T}}
\end{array}\right\rfloor, B=\left[\begin{array}{cc}
0 & 0 \\
0 & 0 \\
0 & 0 \\
0 & 0 \\
\frac{1}{T_{p}} & 0 \\
0 & \frac{1}{T_{T}}
\end{array}\right\rfloor ;
$$

the output vector is chosen as: $y_{e}=\left[\begin{array}{ll}V_{x} & \gamma\end{array}\right]^{\mathrm{T}} ; \gamma=\theta-\alpha=$ $=\theta-\frac{1}{V_{0}} V_{z}$, where $V_{0} \cong V_{x_{0}}$ is the nominal value of the airplane's forward velocity.

To control the longitudinal velocity $V_{x}$ and the slope angle $\gamma$ (to control the angle $\Gamma$ ), one introduces the state vector: $x_{c}=\left[\begin{array}{lll}x_{c_{1}} & x_{c_{2}} & x_{c_{3}}\end{array}\right]^{\mathrm{T}}=\left[\begin{array}{lll}\frac{1}{T \mathrm{~s}+1}\left(\bar{V}_{x}-V_{x}\right) & \frac{V_{0}}{\mathrm{~s}}\left(\gamma_{c}-\gamma\right) & \frac{1}{\mathrm{~s}}(\bar{\Gamma}-\Gamma)\end{array}\right]^{\mathrm{T}}$, where $\bar{V}_{x}, \gamma_{c}, \bar{\Gamma}$ are the imposed values of the variables $V_{x}, \gamma, \Gamma$. According to the last equation, the supplementary vector $x_{c}=\left[\begin{array}{lll}x_{c_{1}} & x_{c_{2}} & x_{c_{3}}\end{array}\right]^{\mathrm{T}}$ depends on the system's state vector, i.e. [14]: $\quad \dot{x}_{c}=A_{21} x+A_{22} x_{c}+B_{21} u_{c}+B_{22} u, \quad$ with $u_{c}=\left[\begin{array}{lll}\bar{V}_{x} & \gamma_{c} & \bar{\Gamma}\end{array}\right]^{\mathrm{T}}$. Taking into account the expression of $y_{e}$ and the last form of (1), one obtains the expressions of $\dot{x}_{c_{1}}, \dot{x}_{c_{2}}, \dot{x}_{c_{3}}$. From these equations and the expression of $\dot{x}_{c}$, it results the matrices:

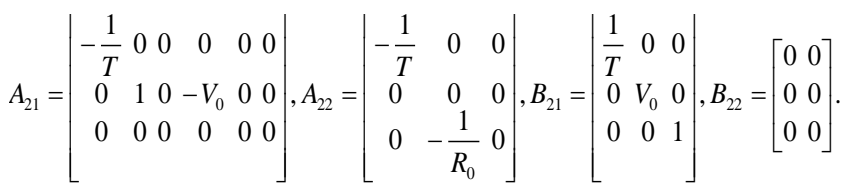

One chooses the following output vector $z=\left[\begin{array}{lll}z_{1} & z_{2} & z_{3}\end{array}\right]^{\mathrm{T}}=$ $=\left[\begin{array}{lllll}c_{1} x_{c_{1}} & c_{2} x_{c_{3}} & c_{3} \delta_{p_{c}} & c_{4} \delta_{T_{c}}\end{array}\right]^{\mathrm{T}}$, where $c_{1}, c_{2}, c_{3}$ are positive constants; the vector $z$ can be written under the form:

$$
z=C_{11} x+C_{12} x_{c}+D_{11} u_{c}+D_{12} u \text {. }
$$

By identification, there are obtained: $C_{11}=0_{4 \times 5}, D_{11}=0_{4 \times 3}$, $C_{12}=\left[\begin{array}{ccc}c_{1} & 0 & 0 \\ 0 & 0 & c_{2} \\ 0 & 0 & 0 \\ 0 & 0 & 0\end{array}\right], D_{12}=\left[\begin{array}{cc}0 & 0 \\ 0 & 0 \\ c_{3} & 0 \\ 0 & c_{4}\end{array}\right\rfloor$. The output vector for achieving the airplane's control in glide slope phase is expressed as:

$$
\begin{aligned}
& y=C_{21} x+C_{22} x_{c}+D_{21} u_{c}+D_{22} u, \\
& C_{21}=\left[\begin{array}{l}
I_{6 \times 6} \\
0_{3 \times 6}
\end{array}\right], C_{22}=\left[\begin{array}{c}
0_{6 \times 3} \\
I_{3 \times 3}
\end{array}\right], D_{21}=0_{9 \times 3}, D_{22}=0_{9 \times 2} .
\end{aligned}
$$

Moreover, the first equation (2) can be put under the form: $\dot{x}=A_{11} x+A_{12} x_{c}+B_{11} u_{c}+B_{12} u$, with $A_{11}=A, A_{12}=0_{6 \times 3}, B_{11}=0_{6 \times 3}$, $B_{12}=B$; putting together the expressions of $\dot{x}, \dot{x}_{c}, z$, and $y$, one obtains the following equation:

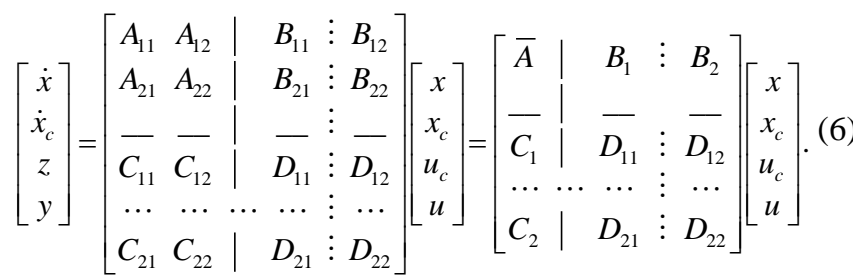

The control law is chosen of the following form: $u=-K y=$ $=-\left[\begin{array}{ll}K_{1} & K_{2}\end{array}\right]\left[\begin{array}{ll}x^{\mathrm{T}} & x_{c}^{\mathrm{T}}\end{array}\right]^{\mathrm{T}}, K=B_{2}^{\mathrm{T}} P_{\infty}$, with $P_{\infty} \in R^{9 \times 9}-$ the solution of the Riccati matriceal equation [13]:

$\bar{A}^{\mathrm{T}} P_{\infty}+P_{\infty} \bar{A}+P_{\infty}\left[\mu^{-2} B_{1} B_{1}^{\mathrm{T}}-B_{2}\left(D_{12}^{\mathrm{T}} D_{12}\right)^{-1} B_{2}^{\mathrm{T}}\right] P_{\infty}+C_{1}^{\mathrm{T}} C_{1}=0$,

where the constant $\mu$ must be minimized, $C_{1}=\left[\begin{array}{ll}C_{11} & C_{12}\end{array}\right]$, $B_{1}=\left[\begin{array}{ll}B_{11}^{\mathrm{T}} & B_{21}^{\mathrm{T}}\end{array}\right]^{\mathrm{T}}, B_{2}=\left[\begin{array}{ll}B_{12}^{\mathrm{T}} & B_{22}^{\mathrm{T}}\end{array}\right]^{\mathrm{T}}$. The block diagram of the subsystem for glide slope phase's control is given in Fig. 2.

For the control of airplane during the flare phase, in the state vector, the altitude $H$ is introduced; the equation associated to this new state is: $\dot{H}=-\frac{1}{\tau} H$. Thus, the new state vector becomes: $x=\left[\begin{array}{lllllll}V_{x} & V_{z} & \omega_{y} & \theta & H & \delta_{p} & \delta_{T}\end{array}\right]^{\mathrm{T}}$; the matrices $A$ and $B$ are modified according to the expressions (3) and to the differential equation of the airplane's altitude. One has chosen as output vector $-y_{e}=\left[\begin{array}{llll}V_{x} & V_{z} & \theta & H\end{array}\right]^{\mathrm{T}}$. The expression of $\dot{H}$ can be written as [13]: $\dot{H}=V_{x_{0}} \theta-V_{z}$; between the two 
equations of $\dot{H}$ there is a difference (error): $e_{\dot{h}}=\dot{H}+\frac{1}{\tau} H$, that must tend to an imposed value $\bar{e}_{\dot{h}}=0$. In order to have $e_{\dot{h}}=\bar{e}_{\dot{h}}$, after the deviation $\tilde{e}_{\dot{h}}=\bar{e}_{\dot{h}}-e_{\dot{h}}$ one will introduce an ideal integrator on the subsystem's direct way; thus, it appears the state: $x_{c_{2}}=\frac{1}{\mathrm{~s}} \tilde{e}_{\dot{h}}$; therefore, $x_{c}=\left[\begin{array}{ll}x_{c_{1}} & x_{c_{2}}\end{array}\right]^{\mathrm{T}}$ and $u_{c}=\left[\begin{array}{ll}\bar{V}_{x} & \bar{e}_{\dot{h}}\end{array}\right]^{\mathrm{T}}$.

As in the glide slope case, one obtains the expressions of $\dot{x}_{c_{1}}, \dot{x}_{c_{2}}$; using these, it results:

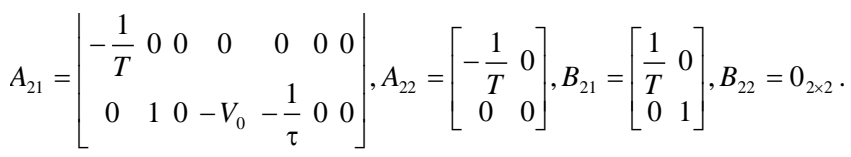

One chooses the output vector $z$ (verifying (4)) of the form:

$$
z=\left[\begin{array}{lll}
z_{1} & z_{2} & z_{3}
\end{array}\right]^{\mathrm{T}}=\left[\begin{array}{llll}
c_{1} x_{c_{1}} & c_{2} x_{c_{2}} & c_{3} \delta_{p_{c}} & c_{4} \delta_{T_{c}}
\end{array}\right]^{\mathrm{T}}
$$

with $C_{11}=0_{4 \times 7}, D_{11}=0_{4 \times 2}, C_{12}=\left[\begin{array}{cc}c_{1} & 0 \\ 0 & c_{2} \\ 0 & 0 \\ 0 & 0\end{array}\right), D_{12}=\left[\begin{array}{cc}0 & 0 \\ 0 & 0 \\ c_{3} & 0 \\ 0 & c_{4}\end{array}\right\rfloor$; the output vector $y$ is: $y=\left[\begin{array}{ll}x^{\mathrm{T}} & x_{c}^{\mathrm{T}}\end{array}\right]^{\mathrm{T}}=\left[\begin{array}{lllllllll}V_{x} & V_{z} & \omega_{y} & \theta & H & \delta_{p} & \delta_{T} & x_{c_{1}} & x_{c_{2}}\end{array}\right]^{\mathrm{T}}$, that can be put under form (5) with $C_{21}=\left\lfloor\begin{array}{l}I_{7 \times 7} \\ 0_{2 \times 7}\end{array}\right\rfloor, C_{22}=\left\lfloor\begin{array}{c}0_{7 \times 2} \\ I_{2 \times 2}\end{array}\right\rfloor$, $D_{21}=0_{9 \times 2}, D_{22}=0_{9 \times 2}$. Also, in this case, $A_{11}=A, A_{12}=0_{7 \times 2}$, $B_{11}=0_{7 \times 2}, B_{12}=B$. The control law is calculated as above. The block diagram of the subsystem for flare phase's control is presented in Fig. 3.

\section{AIRPLANE'S CONTROL IN LATERAL-DIRECTIONAL PLANE}

Before start of the landing two main stages in longitudinal plane, the pilot must cancel the airplane's lateral deviation with respect to the runway (lateral-directional plane); for this, the next subsystem of the new designed ALS will be used [1]; two commands (rudder and ailerons' commands) are necessary.

The state vector and the command vector of the system

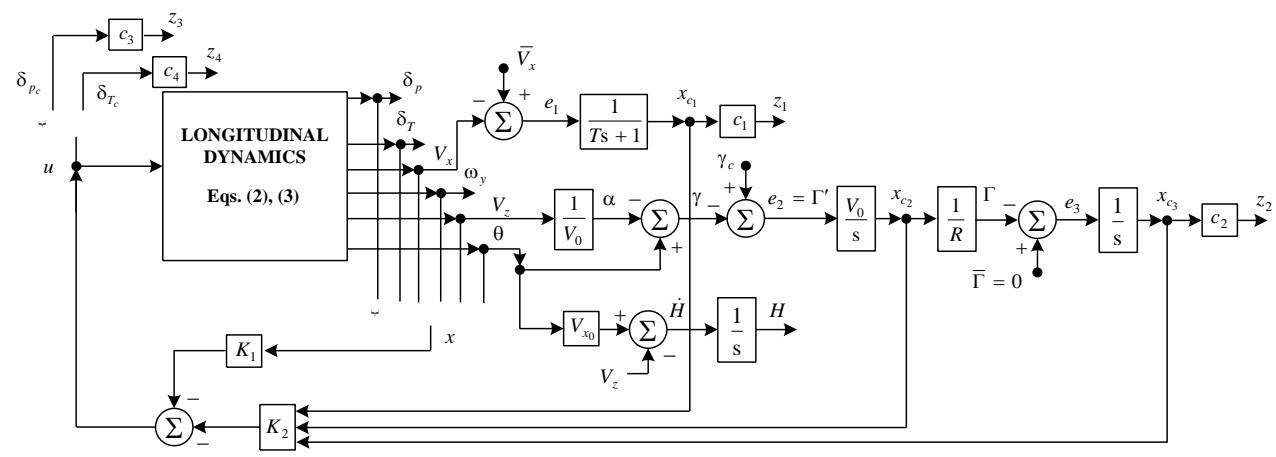

Fig. 2. Airplane control subsystem during glide slope phase (longitudinal plane).

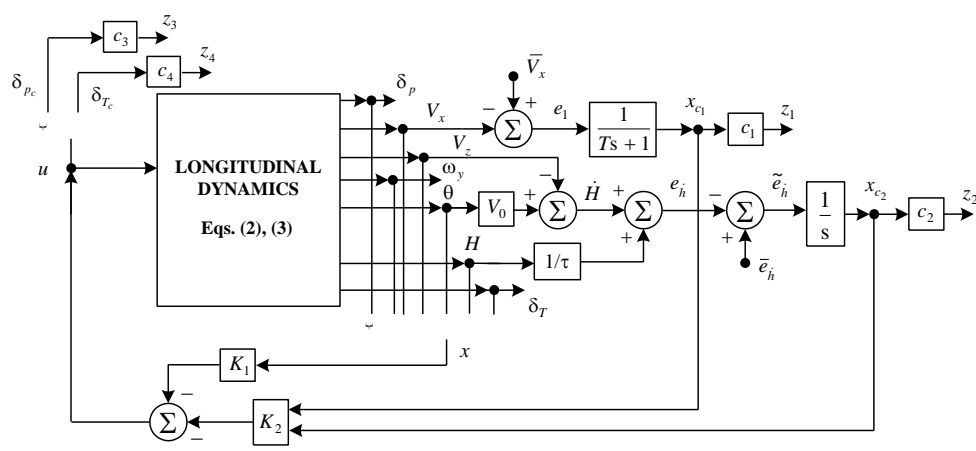

Fig. 3. Airplane control subsystem during flare phase (longitudinal plane).

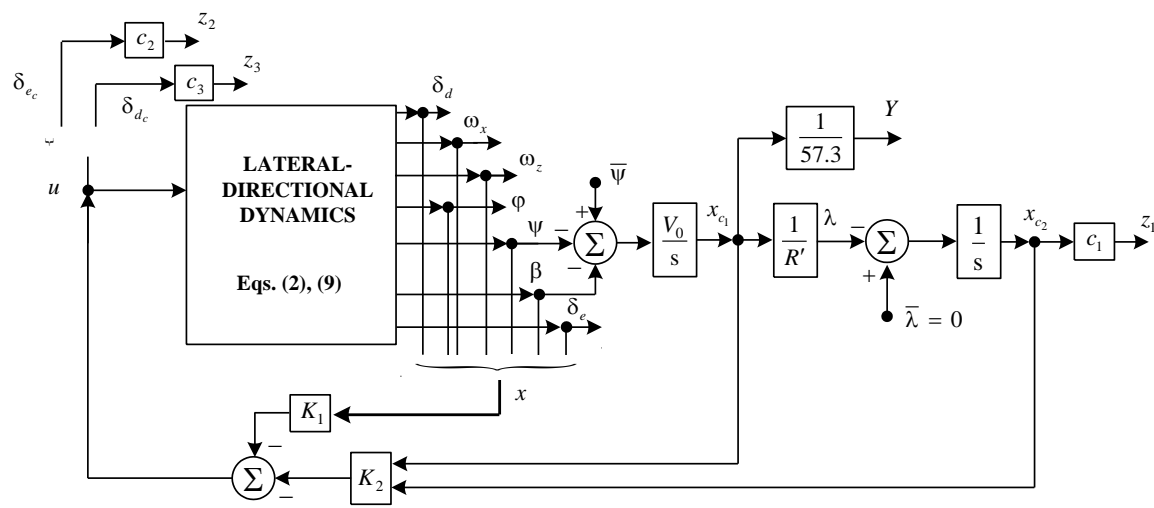

Fig. 4. Airplane control subsystem during initial approach (lateral-directional plane). 
are: $x=\left[\begin{array}{lllllll}\beta & \omega_{x} & \omega_{z} & \varphi & \psi & \delta_{e} & \delta_{d}\end{array}\right]^{\mathrm{T}}, u=\left[\begin{array}{lll}\delta_{e_{c}} & \delta_{d_{c}}\end{array}\right]^{\mathrm{T}}$, where $\delta_{e}$ (ailerons' command) and $\delta_{d}$ (rudder's command) verify the equations: $T_{e} \dot{\delta}_{e}+\delta_{e}=\delta_{e_{c}}, T_{d} \dot{\delta}_{d}+\delta_{d}=\delta_{d_{c}} ; \varphi$ and $\psi$ are the roll and yaw angles, while $\omega_{x}$ and $\omega_{z}$ are the roll and yaw angular rates, respectively. With these, the matrices $A$ and $B$ from airplane's dynamics are, respectively:

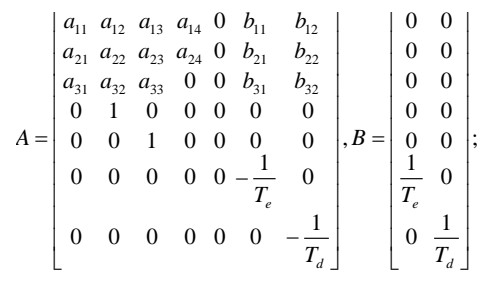

the supplementary state vector is chosen of the form [13]:

$$
x_{c}=\left[\begin{array}{ll}
x_{c_{1}} & x_{c_{2}}
\end{array}\right]^{\mathrm{T}}=\left[\frac{V_{0}}{\mathrm{~s}}(\bar{\psi}-\psi-\beta) \frac{1}{\mathrm{~s}}(\bar{\lambda}-\lambda)\right]^{\mathrm{T}},
$$

such that, in stationary regime, one gets: $\psi+\beta=\bar{\psi}$ and $\lambda=\bar{\lambda}=0$. One obtains the expressions of $\dot{x}_{c_{1}}, \dot{x}_{c_{2}}$; using these, it results: $A_{21}=\left[\begin{array}{ccccccc}-V_{0} & 0 & 0 & 0 & -V_{0} & 0 & 0 \\ 0 & 0 & 0 & 0 & 0 & 0 & 0\end{array}\right], A_{22}=\left[\begin{array}{cc}0 & 0 \\ -\frac{1}{R^{\prime}} & 0\end{array}\right]$, $B_{21}=\left[\begin{array}{cc}V_{0} & 0 \\ 0 & 1\end{array}\right\rfloor, B_{22}=0_{2 \times 2}$. The form of the output vector is $z=\left[\begin{array}{lll}z_{1} & z_{2} & z_{3}\end{array}\right]^{\mathrm{T}}=\left[\begin{array}{llll}c_{1} x_{c_{2}} & c_{2} \delta_{e_{c}} & c_{3} \delta_{d_{c}}\end{array}\right]^{\mathrm{T}} ; \quad z$ verifies (4) with $C_{11}=0_{3 \times 7}, D_{11}=0_{3 \times 2}, C_{12}=\left[\begin{array}{ll}0 & c_{1} \\ 0 & 0 \\ 0 & 0\end{array}\right\rfloor, D_{12}=\left\lfloor\begin{array}{cc}0 & 0 \\ c_{2} & 0 \\ 0 & c_{3}\end{array}\right\rfloor ;$ the vector $y$ for control is: $y=\left[\begin{array}{ll}x^{\mathrm{T}} & x_{c}^{\mathrm{T}}\end{array}\right]^{\mathrm{T}}=\left[\begin{array}{lllllllll}\beta & \omega_{x} & \omega_{z} & \varphi & \psi & \delta_{e} & \delta_{d} & x_{c_{1}} & x_{c_{2}}\end{array}\right]^{\mathrm{T}}$; it verifies the first equation (5) with $C_{21}=\left\lfloor\begin{array}{l}I_{7 \times 7} \\ 0_{2 \times 7}\end{array}\right\rfloor, C_{22}=\left\lfloor\begin{array}{c}0_{7 \times 2} \\ I_{2 \times 2}\end{array}\right\rfloor$,
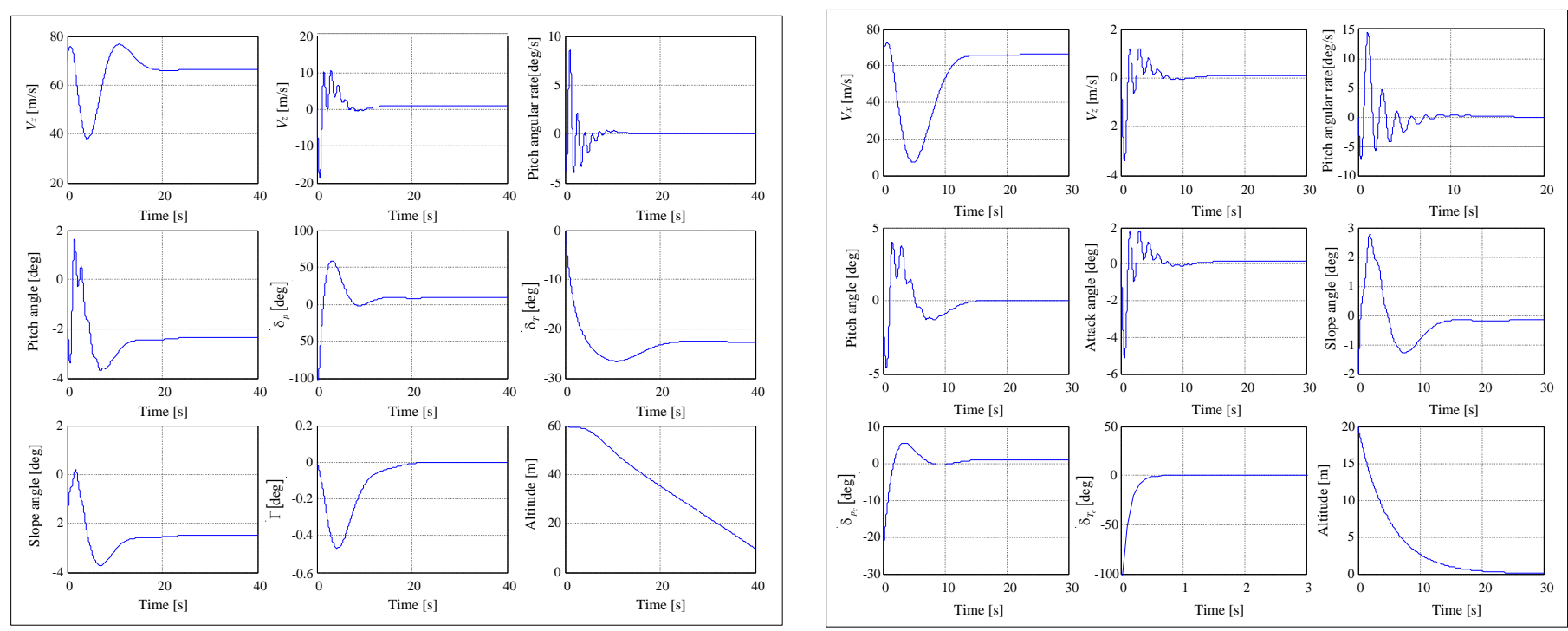

b.

Fig. 5. Time characteristics for the longitudinal plane: a) glide slope phase; b) flare phase. 

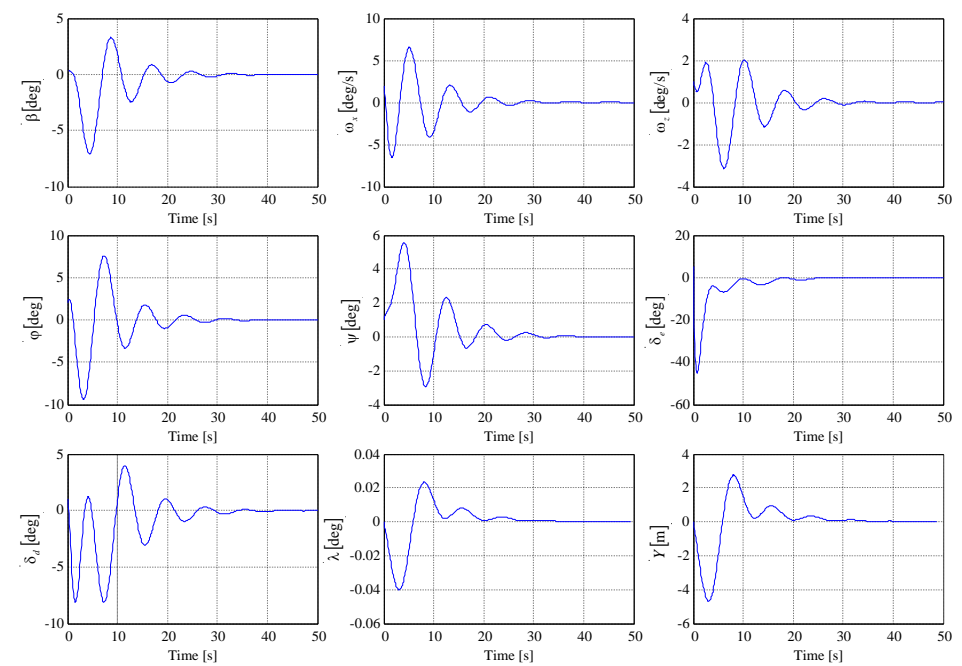

Fig. 6. Time characteristics for the lateral-directional plane.

For lateral-directional plane, the coefficients from Fig. 4 are [15]: $V_{0}=67 \mathrm{~m} / \mathrm{s}, T_{e}=T_{d}=2.1 \mathrm{~s}, c_{1}=650, c_{2}=c_{3}=1, \mu=7,[\bar{\psi} \bar{\lambda}]^{\mathrm{T}}=\left[\begin{array}{ll}0 & 0\end{array}\right]^{\mathrm{T}}$, $x(0)=[0.5 \mathrm{grd} 2 \mathrm{grd} / \mathrm{s} 1 \mathrm{grd} / \mathrm{s} 2 \mathrm{grd} 1 \mathrm{grd} 5 \mathrm{grd} 1 \mathrm{grd}]^{\mathrm{T}}$. The time characteristics associated to the initial approach phase are presented in Fig. 6; it can be observed that the subsystem responds very well and the lateral deviation of the airplane with respect to the runway line is cancelled $(Y \rightarrow 0)$.

\section{CONCLUSION}

In this paper, one designed an ALS using the $\mathrm{H}$-inf technique; it consists of three landing subsystems that have been software implemented, and validated; the first two subsystems are useful for landing control in longitudinal plane (glide slope and flare), while the third one is used in lateral plane. The advantage is the applicability to problems involving multivariate systems with cross-coupling between channels. The whole system has been software implemented, tested, and validated through numerical simulations; promising results have been obtained.

\section{REFERENCES}

[1] R. Lungu and M. Lungu, Airplane Landing Control Using the H-inf Control and the Dynamic Inversion Technique, Intech Publisher, pp. 101-120, 2016.

[2] M. Lungu, Flight Control Systems (Sisteme de Conducere a Zborului), Sitech Publisher, Craiova, 2008.

[3] D. McLean, Automatic Flight Control Systems, Prentice Hall Publisher, 1990.

[4] L. Zhi and W. Yong, "Intelligent landing of unmanned aerial vehicle using hierarchical fuzzy control," IEEE Aerospace Conference, pp. 1-12, 2012.

[5] Y. Ochi and K. Kanai, "Automatic approach and landing for propulsion controlled airplane by $\mathrm{H}$-inf control," IEEE International Conference on Control Applications, Hawaii, pp. 997-1002, 1999.

[6] A. Calise, E. Johnson, M. Johnson, and J. Corban, "Applications of adaptive neural - Networks control to unmanned aerial vehicles," Journal of Harbin Institute of Technology, vol. 38, no. 11, pp. 1865-1869, 2006.

[7] T. Wagner and J. Valasek, "Digital Autoland Control Laws Using Quantitative Feedback Theory and Direct Digital Design", Journal of Guidance, Control, and Dynamics, vol. 30, no. 5, pp. 1399-1413, 2007.

[8] J. Jang and G. Jeon, "A parallel neuro-controller for DC motors containing nonlinear friction," Neurocomputing, vol. 30, pp. 233-248, 2000.

[9] S. Seshagiri and H. K. Khalil, "Output feedback control of nonlinear systems using RBF neural network," IEEE Transactions on Neural Networks, vol. 11, pp. 69-79, 2000.
[10] B. Parkinson, M. O'Connor, and K. Fitzgibbon, "Airplane automatic approach and landing using GPS," Global Positioning System: Theory and Applications, vol. II, pp. 397-425, 1996.

[11] R. Lungu and M. Lungu, "Application of $\mathrm{H}_{2} / \mathrm{H}_{\infty}$ and dynamic inversion techniques to airplane landing control," Aerospace Science and Technology, vol. 46, no. 1, pp. 146-158, 2015.

[12] J. Juang, H. Chang, and W. Chang, "Intelligent automatic landing system using time delay neural network controller," Applied Artificial Intelligence: An International Journal, vol. 17, no. 7, pp. 563-581, 2003.

[13] R. Lungu and M. lungu, "Airplane automatic control during landing (Controlul automat al aeronavelor la aterizare). Sitech Publisher, 2015.

[14] O. Yashimaso and K. Kimio, "Automatic Approach and Landing for propulsion controlled airplane by H-inf control," in Proc. the IEEE International Conference on Control Applications, Hawaii, pp. 997-1002, 1999.

[15] R. Lungu, M., Lungu, and T. L. Grigorie, "ALSs with conventional and fuzzy controllers considering wind shears and gyro errors," Journal of Aerospace Engineering, vol. 26, no. 4, pp. 794-813, 2012.

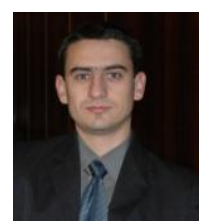

Mihai Lungu was born in Craiova, Romania, in 1980. In 2004 he graduated the University of Craiova, Faculty of Electrical Engineering, Specia- lization: Board Equipments and Devices. In 2008 he received the Ph.D. degree in the domain of aerospace engineering (Ph.D. thesis: complex adaptive and optimal systems for the Stabilization, Navigation and Control of the Flying Objects). Since 2016 he is an associate professor at the University of Craiova, Electrical, Energetic, and Aerospace Engineering Department. His fields of interest include: the flight control systems, automatic pilots, gyroscopic equipment and systems etc. His scientific experience includes: 7 books/chapters in books; 2 laboratory guidelines; 1 patent; 140 published papers; 17 scientific research grants; 138 citations.

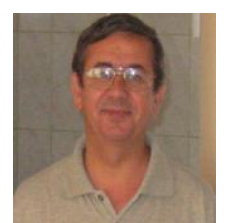

Romulus Lungu was born in Bistrita Nasaud, Romania, in 1952. In 1976 he graduated the University of Craiova, Faculty of Electrotechnics, specialization: automatics and computers. In 1991 he received the $\mathrm{Ph} . \mathrm{D}$. degree (aerospace engineering domain). Since 1997 he is a professor at the University of Craiova, Avionics Division. He is the founder of the specialisation board equipments and devices at the University of Craiova and since 2009 he is Ph.D. leader in the domain of aerospace engineering. His interest fields are: automatics of the flying objects, rockets' control, auto-pilots, gyroscopic equipment and systems etc. His scientific experience includes: 13 books/chapters in books; 6 laboratory guidelines; 237 published papers; 22 scientific research grants; 152 citations. 